heavy chains. A similar association (with the human Gm allotypes) has been reported in MG (Nakao et al. Lancet i, 677; 1980). These findings might explain the generally poor correlation between the total anti-AChR titre and disease severity. Clearly, specific subpopulations of antibodies may also be important in MG and will be defined when human monoclonal anti-AChR is available.

In EAMG several workers have used monoclonal antibodies produced by hybridomas to analyse the anti-AChR. A single monoclonal antibody reactive with mammalian AChR can passively transfer EAMG to mice (J. Lindstrom, Salk Institute) or rats (V. Lennon, Mayo Clinic). Surprisingly, antibodies raised in inbred rats against each of three distinct monoclonal antibodies inhibited the activity of the others suggesting that they shared a common idiotype (the antigenspecific, hypervariable region) (V. Lennon). However, each anti-idiotype antibody inhibited only a proportion of the anti-AChR in polyclonal EAMG sera and when anti-idiotype-producing animals were immunized directly against $\mathrm{AChR}$ they still developed electrophysiological evidence of EAMG.

J. Lindstrom has used monoclonal antibodies rasied against intact $\mathrm{AChR}$ and its polypeptide subunits to map determinants on receptor extracted from many different species. The main immunogenic region appears to be on the $\alpha$-subunit of Torpedo and eel AChR and similar determinants are found on AChR from mammals including humans. Three monoclonal antibodies reacting with $\alpha, \beta$ and $\gamma$ subunits can bind to human AChR and inhibit the binding of a proportion of human anti-AChR (S. Tzartos, Salk Institute). These results are important because they suggest that the determinants to which human anti-AChR bind are more restricted than was previously inferred from the heterogeneous characteristics of MG anti-AChR (as defined by variability in $x$ and $\lambda$ light chains, binding to the $\alpha$-bungarotoxin binding site, crossreactivity with other $A C h R$ preparations).
Moreover, using a polyclonal anti-idiotype serum raised in rabbits against human antiAChR, A. K. Lefvert (Karolinska Institute) has found some sharing of antiAChR idiotypes between different MG patients.

The treatment of MG has improved since the autoimmune basis for the disease has been widely recognized. Immunosuppressants are used much more freely with good results, and plasma exchange can produce marked temporary improvement. Although several interesting experimental approaches to treatment, including immunization against modified $\mathrm{AChR}$ and anti-idiotype induction in rabbits with EAMG (S. Fuchs), have met with some success, it will be some time before any can be tried in the human disease. Nevertheless, if there is less heterogeneity and greater idiotype restriction in MG antiAChR than previously thought, and if certain idiotypes can be shown to play a crucial role in the disease process, specific immunotherapy may become a realistic goal.

\title{
Disposing of dioxins by oxidation
}

\section{from Alastair Hay}

DISPOSAL of waste contaminated by polychlorinated dibenzodioxins (PCDDs) has long been a problem for sections of the chemical industry. Toxic and often remarkably stable, the PCDDs are produced as unwanted by-products during the manufacture of chlorinated phenols.

The best known and most extensively studied PCDD is 2,3,7,8-tetrachlorodibenzo- $p$-dioxin (TCDD). It is produced during the manufacture of 2,4,5-trichlorophenol - the starting point for the widely used herbicide 2,4,5-trichlorophenoxyacetic acid (2,4,5-T).

Although pure TCDD is very stable (a temperature in excess of $800^{\circ} \mathrm{C}$ is required for its thermal degradation), if dissolved in organic solvents such as methanol or benzene it is exceptionally unstable in the presence of ultraviolet light. The solvents act as hydrogen donors, replacing aromatic ring chlorines with hydrogen as TCDD is photolytically degraded. The reaction sequence proceeds in stages with only one chlorine atom being replaced at any one time. Eventually TCDD is degraded to the unchlorinated parent dibenzodioxin (D.G. Crosby et al. Science 173, 748; 1971). Many orders of magnitude less toxic than TCDD, dibenzodioxin too is decomposed by light (J.R. Plimmer et al. Adv. Chem. 120, 44; 1973).

The higher chlorinated dibenzodioxins, hexa-, hepta- and octachlorodibenzo- $p$ dioxin, produced in the manufacture of the wood preservative pentachlorophenol, are also subject to photolytic degradation (C. Rappe et al. Ann. N.Y. Acad. Sci. 320, 1;
1979).

Under field conditions TCDD can be subjected to photolytic degradation by sunlight when a suitable solvent/hydrogen donor is present. When a preparation of the herbicide 'Agent Orange' - a formulation of $2,4,5-\mathrm{T}$ containing 15 p.p.m. TCDD - was exposed to sunlight, the dioxin was rapidly degraded with less than half remaining after 6 hours (A.S. Wong and D.E. Crosby in Dioxin: Toxicological and Chemical Aspects (eds Cattabeni, F. et al., Spectrum, New York; 1978). When TCDD was dissolved in an oil/emulsifier mixture and exposed to sunlight, complete degradation occurred within 2 days (A.S. Wong and C.G. Crosby op. cit.).

In industry, photodegradation of PCDDs is not very practical and most manufacturers dispose of the chemicals by high-temperature incineration. A method of degradation using chemical processes might provide a more attractive option and in this issue of Nature (p.323), D.C. Ayres reports that $\mathrm{PCDDs}$ can be degraded efficiently by an oxidative procedure involving ruthenium tetroxide.

Ruthenium tetroxide is a powerful oxidizing agent which reacts with a wide range of organic substances, and according to Ayres, it can be used in solution in water or in organic solvents with no nucleophilic character, such as chloroform, nitro-

Alastair Hay is in the Department of Chemical Pathology at Leeds University. methane or carbon tetrachloride. Ayres reports that 2,7-dichlorodibenzo-p-dioxin (DCDD) was destroyed by ruthenium tetroxide in carbon tetrachloride solution when the cocktail was allowed to stand overnight at ambient temperature. The rate of oxidation increases with increase in temperature: at $30^{\circ} \mathrm{C} \mathrm{DCDD} \mathrm{has} \mathrm{a} \mathrm{half} \mathrm{life}$ of $215 \mathrm{~min}$ whilst at $50^{\circ} \mathrm{C}$, the half life falls to $38 \mathrm{~min}$. The oxidation of TCDD is a slower process: at $20^{\circ} \mathrm{C}$ this chemical has a half life of $560 \mathrm{~min}$ in the ruthenium tetroxide mixture. At $70^{\circ} \mathrm{C}$, however, the half life plummets to a mere $15 \mathrm{~min}$.

Ayres believes that his results demonstrate that ruthenium tetroxide can be safely used for the detoxification of glassware and the periodical purging of industrial reactors to counteract the accumulation of PCDD residues. The process need not be expensive, Ayres claims, for ruthenium tetroxide need only be used in catalytic amounts after which it can be regenerated using a consumable secondary oxidant, such as hypochlorite.

Although Ayres does not know the nature of the fragments formed during the oxidation of the PCDDs, he reports that chlorophenols subjected to ruthenium oxidation undergo extensive decomposition to yield chloride ions and no significant levels of organic products. It remains to be seen whether this is the fate of the PCDDs, but it is clear that oxidation must now challenge incineration as the method of choice for disposing of unwanted polychlorinated dibenzo- $p$-dioxins in industry. 